\title{
Time-Frequency Distributions for Automatic Speech Recognition
}

\author{
Alexandros Potamianos, Member, IEEE, and Petros Maragos, Fellow, IEEE
}

\begin{abstract}
The use of general time-frequency distributions as features for automatic speech recognition (ASR) is discussed in the context of hidden Markov classifiers. Short-time averages of quadratic operators, e.g., energy spectrum, generalized first spectral moments, and short-time averages of the instantaneous frequency, are compared to the standard front end features, and applied to ASR. Theoretical and experimental results indicate a close relationship among these feature sets.
\end{abstract}

Index Terms-Speech analysis, speech processing, speech recognition, time-frequency analysis.

\section{INTRODUCTION}

$\mathbf{T}$ IME-FREQUENCY distributions and short-time averages of quadratic operators are very popular front-end features for automatic speech recognition (ASR). Indeed, the "standard" front-end feature set is the inverse cosine transformation of the short-time-frequency energy distribution. Despite the standardization of the ASR front-end, there has been a significant amount of research on using alternate time-frequency distributions as (possibly additional) ASR features. A good review of such efforts can be found in [7]. However, such efforts are often lacking in theoretical or experimental justification. In this paper, we attempt to outline the relationships between some popular alternative feature sets and the "standard" front-end features, and to present experimental ASR evidence that supports these claims. We hope that this study will help guide future ASR front-end research.

The following two types of nonparametric features are investigated in this paper: i) short-time averages of quadratic operators, e.g., energy spectrum [8], ii) generalized first spectral moments and weighted short-time averages of the instantaneous frequency. Note that the standard feature set is included in the first family of time-frequency distributions. Our goal is to show (both theoretically and experimentally) a close relationship among these feature sets and the standard feature set.

Manuscript received December 8, 1999; revised June 22, 2000. This work was supported in part by the U.S. National Science Foundation under Grants MIP-9396301 and MIP-9421677. The work of P. Maragos was supported by the Greek G.S.R.T. program in Language Technology under Grant 98GT26.

A. Potamianos was with the School of Electrical and Computer Engineering, Georgia Institute of Technology, Atlanta, GA 30332 USA. He is now with Bell Laboratories, Lucent Technologies, Murray Hill, NJ 07074 USA (e-mail: potam@ research.bell-labs.com).

P. Maragos was with the School of Electrical and Computer Engineering, Georgia Institute of Technology, Atlanta, GA 30332 USA. He is now with the Department of Electrical and Computer Engineering, National Technical University of Athens, Zografou, 15773 Athens, Greece.

Publisher Item Identifier S 1063-6676(01)01667-4.
The organization of the paper is as follows: First, we introduce the energy operator and the energy spectrum, and compare it to other spectral envelope representations. In Section III, short-time instantaneous frequency estimators are proposed in the context of the AM-FM modulation model, the sinusoidal model, and spectral estimation. The estimators are compared to the spectral envelope and their merits as ASR features are discussed. Finally, experimental ASR results are given in Section IV. The authors assume in the presentation some familiarity with the sinusoidal speech model [5], the AM-FM modulation model [3] and energy operators [2], [4].

\section{QUADRATIC OPERATORS AND ENERGY SPECTRUM}

The energy operator is defined for continuous-time signals $x(t)$ as

$$
\Psi_{c}[x(t)] \triangleq[\dot{x}(t)]^{2}-x(t) \ddot{x}(t)
$$

where $\dot{x}=d x / d t$. Its counterpart for discrete-time signals $x(n)$ is

$$
\Psi_{d}[x(n)] \triangleq x^{2}(n)-x(n-1) x(n+1) .
$$

The nonlinear operators $\Psi_{c}$ and $\Psi_{d}$ were developed by Teager during his work on speech production modeling [11] and were first introduced systematically by Kaiser [2]. When $\Psi_{c}$ is applied to signals produced by a simple harmonic oscillator, e.g., a mass-spring oscillator, it can track the oscillator's energy (per half unit mass), which is equal to the squared product of the oscillation amplitude and frequency; thus the term energy operator. The energy operator has been applied successfully to demodulation and has many attractive features such as simplicity, efficiency, and adaptability to instantaneous signal variations [3]. The attractive physical interpretation of the energy operator has led to its use as an ASR feature extractor in various forms, see for example [12], [13].

The energy spectrum, introduced in [8], is a general timefrequency distribution based on the energy operator. Assume that $x(n)$ is filtered by a bank of $K$ bandpass filters centered at frequencies $\omega_{k}$ to obtain $K$ band-passed signals: $x_{k}(n), k=$ $1 \cdots K$. The following time and frequency relations hold

$$
x_{k}(n)=x(n) * h_{k}(n) \leftrightarrow X_{k}(\omega)=X(\omega) H_{k}(\omega)
$$

where $h_{k}(n)$ is the impulse response and $H_{k}(\omega)$ is the frequency response of the $k$ th filter and $n$ is the discrete-time sample index. The energy spectrum $\operatorname{ES}(n, k)$ is defined as the 


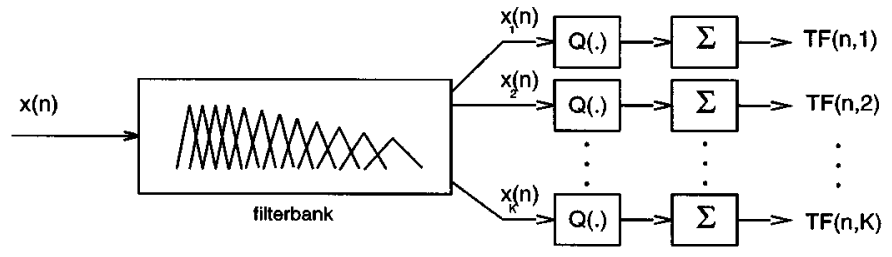

Fig. 1. Time-domain implementation of filterbank ASR front-end.

short-time average of the energy operator applied to the family of band-passed signals $x_{k}(n)$, i.e.,

$$
E S(n, k)=\sum_{m=n}^{n+N-1} \Psi_{d}\left[x_{k}(m)\right]
$$

where $N$ is the length of the short-time averaging window (in samples). Using Parseval's relation one can show

$$
\begin{aligned}
& \sum_{n=-\infty}^{\infty} x(n+a) x(n+b) \\
& \quad=\frac{1}{2 \pi} \int_{-\pi}^{\pi} \cos [(a-b) \omega]|X(\omega)|^{2} d \omega
\end{aligned}
$$

assuming that $x(n)$ is real. Thus

$$
\sum_{n=-\infty}^{\infty} \Psi_{d}[x(n)]=\frac{1}{2 \pi} \int_{-\pi}^{\pi}[1-\cos (2 \omega)]|X(\omega)|^{2} d \omega
$$

Assuming that $x(n)$ is zero outside of the window $[n, n+N-1]$ the energy spectrum can be expressed as

$$
E S(n, k)=\frac{1}{2 \pi} \int_{-\pi}^{\pi}[1-\cos (2 \omega)]\left|H_{k}(\omega) X(\omega)\right|^{2} d \omega
$$

In Fig. 1, the time-domain implementation of a general filterbank-based ASR front-end is shown. Following the notation introduced above $x(n)$ is filtered by a bank of $K$ filters. The feature set at time index $n\{T F(n, k): k=1 \cdots K\}$ is defined as the short-time average of the output of a quadratic operator $Q(\cdot)$ applied to each one of the band-passed signals $x_{k}(n)$, i.e.,

$$
T F(n, k)=\sum_{m=n}^{n+N-1} Q\left[x_{k}(m)\right] .
$$

The general form of the quadratic operator is

$$
Q[x(n)]=\sum_{m} \sum_{r} c_{m r} x(n+m) x(n+r)
$$

where $c_{m r}$ are constants. For $Q=\Psi_{d}$ the time-frequency distribution obtained in Fig. 1 is the energy spectrum: $T F(n, k)=E S(n, k)$. For $Q(x)=x^{2}$ the time-frequency distribution obtained is the short-time smooth power spectral envelope $^{1} T F(n, k)=P S(n, k)$ where

$$
P S(n, k)=\sum_{m=n}^{n+N-1}\left[x_{k}(m)\right]^{2}=\frac{1}{2 \pi} \int_{-\pi}^{\pi}\left|H_{k}(\omega) X(\omega)\right|^{2} d \omega
$$

${ }^{1}$ For computational efficiency the spectral envelope $P S(n, k)$ is computed as $S(n, k)=(1 / \pi) \int_{0}^{\pi}\left|X_{k}(\omega)\right|^{2} d \omega$ rather than in the time domain as in Fig. 1.

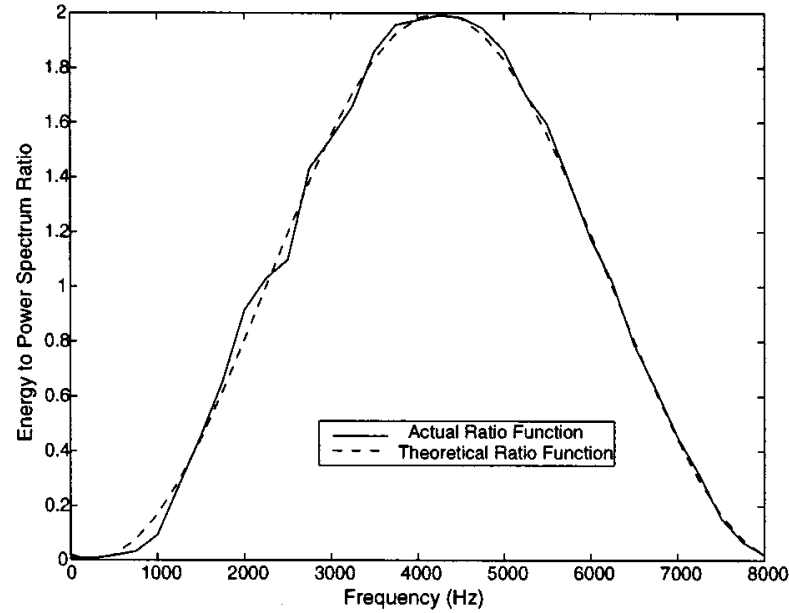

Fig. 2. Ratio of energy spectrum over power spectral envelope.

assuming $x(n)$ is zero outside $[n, n+N-1]$.

Using (7) and (10), the ratio between the power spectral envelope and the energy spectrum can be approximated by

$$
\frac{E S(n, k)}{P S(n, k)} \approx 1-\cos \left(2 \omega_{k}\right)=2 \sin ^{2}\left(\omega_{k}\right)
$$

The approximation is valid for narrowband signals $x_{k}(n)$, where the spectral energy $\left|X_{k}(\omega)\right|$ is concentrated around $\omega_{k}$ and the slowly-varying (in frequency) $\cos (2 \omega)$ term can be assumed constant within the bandwidth of $x_{k}(n)$. Second-order approximations of (7), i.e., $E S(n, k)=(1 / \pi) \int_{-\pi}^{\pi} \omega^{2}\left|H_{k}(\omega) X(\omega)\right|^{2} d \omega$, can be shown to cause formant spectral peak translation in addition to the scaling apparent in (11). Specifically, formant peaks with center frequencies up to $F_{s} / 4 \mathrm{~Hz}$ are translated toward the lower frequencies in the energy spectrum, and vice-versa for formant frequencies higher than $F_{s} / 4$ (thus formant translation is a function of the sampling frequency $F_{s}$ ).

In Fig. 2, a time-slice of the ratio $\{E S(n, k) / P S(n, k): k=$ $1 \cdots K\}$ is shown (solid line) together with the function $1-$ $\cos \left(2 \omega_{k}\right)$ (dashed line). The ratio is computed for a single $20 \mathrm{~ms}$ speech frame of the vowel /ih/. A uniformly-spaced Gabor filterbank with $250-\mathrm{Hz} 3-\mathrm{dB}$ bandwidth per Gabor filter was used for computing ES and PS (sampling frequency $16 \mathrm{kHz}$ ). Differences between the computed and predicted ratio values are due to second-order effects (ripples in Fig. 2 correspond to formant translations in $E S$ ) and to the use of the (approximate) discrete Fourier transform instead of the discrete-time Fourier transform. Most ASR front-ends use the inverse cosine transform of the logarithm of $P S(n, k)$ as a feature set (cepstrum). In the cepstrum domain, the difference between energy cepstrum and "standard" cepstrum is approximately a time-independent bias.

In general, using (5) the sum of any quadratic operator $Q[x(n)]$ output (e.g., see [4], [1]) can be expressed as

$$
\sum_{n=-\infty}^{\infty} Q[x(n)]=\sum_{\ell=0}^{L} \frac{c_{\ell}}{\pi} \int_{-\pi}^{\pi} \cos (\ell)|X(\omega)|^{2} d \omega
$$


where $c_{\ell}$ are arbitrary constants. For narrowband signals $x_{k}(n)$, $\cos (\ell \omega)$ can be assumed constant around $\omega_{k}$ and the short-time average of $Q\left[x_{k}(n]\right.$ can be expressed as

$$
\log [T F(n, k)]-\log [P S(n, k)] \approx b(k)
$$

i.e., the difference between the log of any time-frequency distribution produced by the generalized ASR front-end in Fig. 1 and the log of the power spectral envelope is approximately a time-independent bias vector $b$ (also in the cepstrum domain).

Given the similarity between the time-frequency distributions of quadratic operators it is expected that ASR performance will also be similar for various front-ends that use short-time averages of quadratic operators as features. However, as the size of the short-time window $N$ decreases and/or the bandwidth of the filter $H_{k}(\omega)$ increases the differences among $\operatorname{TF}(n, k)$ are no longer time-invariant, i.e., $b=b(n, k)$ and significant ASR performance differences may arise between various front-ends (see for example [12] where the energy operator is applied to the unfiltered signal). The equivalence between $\operatorname{ES}(n, k), P S(n, k)$ and $S(n, k)=(1 / \pi) \int_{0}^{\pi}\left|X_{k}(\omega)\right|^{2} d \omega$ as features (in the cepstrum domain) for ASR is experimentally shown in Section IV.

\section{Spectral Moments AND AVERage Instantaneous FREQUENCY}

In this section, we investigate the relation between various time-frequency distributions motivated by the AM-FM modulation model [3], the sinusoidal speech model [5], and spectral analysis. The distributions compute the short-time instantaneous frequency in different frequency bands. The distributions are compared to the short-time spectral envelope and their application to ASR is discussed.

The AM-FM modulation model, introduced in [3], describes a speech resonance as a signal with a combined amplitude modulation (AM) and frequency modulation (FM) structure

$$
r(t)=a^{I}(t) \cos \left(2 \pi\left[f_{c} t+\int_{0}^{t} q(\tau) d \tau\right]+\theta\right)
$$

where

$f_{c} \triangleq F \quad$ "center value" of the formant frequency;

$q(t) \quad$ frequency modulating signal;

$a^{I}(t) \quad$ time-varying amplitude.

The instantaneous formant frequency signal is defined as $f^{I}(t)=f_{c}+q(t)$. The speech signal $x(t)$ is modeled as the sum $x(t)=\sum_{k=1}^{K} r_{k}(t)$ of $K$ such AM-FM signals, one for each formant. A general family of time-frequency distributions of amplitude weighted short-time averages of the instantaneous frequency is defined as

$$
T F_{1}(n, k)=\frac{\sum_{m=n}^{n+N-1}\left[a_{k}^{I}(n)\right]^{\gamma} f^{I}(n)}{\sum_{m=n}^{n+N-1}\left[a_{k}^{I}(n)\right]^{\gamma}}
$$

where $a_{k}^{I}(n), f_{k}^{I}(n)$ are the amplitude envelope and the instantaneous frequency, respectively, of the narrow-band signal $x_{k}(n)$ in (3), and $\gamma$ is an arbitrary constant. Note that $T F_{1}$ for $\gamma=0$, $F_{u}=\left\langle f^{I}(t)\right\rangle$, was used for fundamental frequency estimation in [10] and $T F_{1}$ for $\gamma=2, F_{w}=\left\langle\left[a^{I}(t)\right]^{2}\left[f^{I}(t)\right]\right\rangle /\left\langle\left[a^{I}(t)\right]^{2}\right\rangle$, (also referred to as the "pyknogram") was used for formant tracking in [9].

The sinusoidal model [5] models the speech signal $x(t)$ as a superposition of short-time varying sinusoids. Similarly the narrow-band signals $x_{k}(t)$ can be modeled using a sinusoidal model as

$$
x_{k}(t)=\sum_{p=1}^{P_{k}} a_{k p} \sin \left(2 \pi f_{k p} t+\theta_{k p}\right)
$$

where $a_{k p}, f_{k p}, \theta_{k p}$ are the constant (in an analysis frame $[n, n+N-1])$ amplitudes, frequencies, and phases, respectively, of the $p=1 \cdots P_{k}$ sinusoids modeling $x_{k}(t)$. A general time-frequency representation can be obtained as a weighted average of $f_{k p}$ as follows:

$$
T F_{2}(n, k)=\frac{\sum_{p=1}^{P_{k}}\left[a_{k p}(n)\right]^{\gamma} f_{k p}(n)}{\sum_{p=n}^{P_{k}}\left[a_{k p}(n)\right]^{\gamma}}
$$

where $\gamma$ is an arbitrary constant. Note that the summation index $p$ is a frequency index.

Finally a third type of time-frequency distribution is the generalized first spectral moment

$$
T F_{3}(n, k)=\frac{1}{2 \pi} \frac{\int_{0}^{\pi}\left|X_{k}(\omega)\right|^{\gamma} \omega d \omega}{\int_{0}^{\pi}\left|X_{k}(\omega)\right|^{\gamma} d \omega}
$$

where $\gamma$ is an arbitrary constant. Note that $T F_{3}$ for $\gamma=0.5$ has been used as an ASR feature in [6]. Next we investigate the relationships among the three time-frequency distributions $T F_{1}, T F_{2}$, and $T F_{3}$ defined above.

Clearly $T F_{2}$ is a short-time estimate of the generalized spectral moment, i.e., $T F_{3} \approx T F_{2}$. As $P_{k}$ goes to infinity in (16) (i.e., more sinusoidal components are included in the approximation) the time-frequency representations $T F_{2}, T F_{3}$ become equal. The relation between $T F_{1}$ and $T F_{2}$ is more complicated and depends on the value of the amplitude weight $\gamma$. Specifically, for $\gamma=2$, it is easy to show that all three time-frequency distributions are equivalent, i.e., $T F_{1}=T F_{2}=T F_{3}$ [9]. For $\gamma \neq 2$, one can show (along the lines of the proof for $\gamma=0$ in [10]) that under the assumption that $f_{k p p}$ are harmonically related

$$
T F_{1}(n, k) \approx \frac{\left[a_{k M}(n)\right]^{2} f_{k M}(n)+\frac{\gamma}{2} \sum_{p \neq M}\left[a_{k p}(n)\right]^{2} f_{k p}(n)}{\left[a_{k M}(n)\right]^{2}+\frac{\gamma}{2} \sum_{p \neq M}\left[a_{k p}(n)\right]^{2}}
$$

where $a_{k M}=\max _{p}\left\{a_{k p}\right\}$ is the amplitude of the sinusoid $f_{k M}$ with the greatest amplitude. Thus, we have established that 
$T F_{1}, T F_{2}, T F_{3}$ are equivalent for $\gamma$ around 2. Next, we investigate the relationship between $T F_{3}$ and the standard ASR front-end.

The standard ASR front-end computes the short-time spectral energy in each of the frequency bins $k$ as follows: $S(n, k)=(1 / \pi) \int_{0}^{\pi}\left|X_{k}(\omega)\right|^{2} d \omega$, where $X_{k}(\omega)$ is defined in (3). Assuming that $h_{k}(n)$ in (3) is the real Gabor filter's impulse response, the frequency response can be expressed as

$$
H_{k}(\omega)=(\sqrt{\pi} / 2 \alpha)\left(e^{-\left(\omega-\omega_{k}\right)^{2} / 4 \alpha^{2}}+e^{-\left(\omega+\omega_{k}\right)^{2} / 4 \alpha^{2}}\right)
$$

where $\alpha$ is proportional to the bandwidth of the filter. For $\gamma=2$ and for a Gabor filterbank, the spectral moment time-frequency distribution $T F_{3}$ can be expressed as a function of the standard front-end feature set $S$ as follows ${ }^{2}$

$$
T F_{3}(n, k) \approx \omega_{k}+\frac{\alpha^{2}}{S(n, k)} \frac{d S(n, k)}{d \omega_{k}}
$$

where $d S(n, k) / d \omega_{k}$ is the derivative of the short-time spectral energy distribution $S$ with respect to the center frequency of the filterbank filter $\omega_{k}$.

Given the close relationship between $T F_{3}$ and $S$ it might be expected that both distributions will perform similarly when used as features for ASR. However, $S$ is a zeroth-order spectral estimator while $T F_{3}$ is a first-order one [see (18)]. Thus, $T F_{3}$ is expected to be a less robust estimator and have inferior classification performance. Indeed, we have experimentally verified that the separability of phonemic classes in the $S(k)$ space is significantly better than in the $T F_{3}(k)$ space. Efforts to augment the standard feature set $S$ by one of $T F_{1-3}$ are expected to have little success [6] due to the high correlation between the two feature sets exemplified by (21). Note, however, that gains may be observed when different analysis time-scales are used for the two distributions or for mismatched ASR conditions (in training and testing), e.g., noisy speech. Further, since $T F_{1} \approx T F_{3} \approx T F_{2}$ for $\gamma \approx 2$ the above statements are also valid for $T F_{1}$ and $T F_{2}$.

\section{EXPERIMENTS}

In this section, the recognition accuracy of the various feature sets is compared for a connected digit recognition task. ${ }^{3}$ A hidden Markov model (HMM) recognizer was used with eight Gaussian mixtures per HMM state. Each digit was modeled by a left to right HMM unit, 8-10 states in length. The test set consists of 4304 digit strings (13 185 digits) collected over the public switched telephone network.

The front-ends evaluated were (all with $20 \mathrm{~ms}$ analysis window, $10 \mathrm{~ms}$ update, and identical filterbank spacing and bandwidths)

1) "standard" mel-filterbank front-end $S(n, k)$ using triangular filters;

2) mel-filterbank front-end using Gaussian filters $P S(n, k)$;

3) energy spectrum $E S(n, k)$;

${ }^{2}$ The approximation error is greatest for $\omega_{k}$ close to 0 and for large values of bandwidth parameter $\alpha$.

${ }^{3}$ Similar results were obtained on the TIMIT phone recognition task.
TABLE I

DIGIT ERROR RATE FOR DIFFERENT TIME-FREQUENCY DISTRIBUTIONS AS ASR FEATURE SETS $\left(C^{-1}\right.$ IS THE INVERSE Cosine TRANSFORM)

\begin{tabular}{l|l|l}
\hline Front End & Fltbnk & W.Acc \\
\hline \hline Baseline cep: $C^{-1}\{S\}=c_{1} . . c_{12}$ & triang. & $97.1 \%$ \\
Power cep: $C^{-1}\{P S\}$ & Gabor & $96.8 \%$ \\
Energy cep: $C^{-1}\{E S\}$ & Gabor & $97.0 \%$ \\
Pyknogram cep: $C^{-1}\left\{T F_{1}\right\}$ & Gabor & $81.1 \%$ \\
\hline
\end{tabular}

4) amplitude weighted average instantaneous frequency $T F_{1}(n, k)$ for $\gamma=2$.

For all front ends the feature set consisted of the mean square of the signal ("standard energy"), the inverse cosine of the above described time-frequency distributions (cepstrum), and the first and second derivatives of these features.

The results are shown in Table I. As expected the performance of $S, P S$ and $E S$ is very similar, while $T F_{1}$ performs significantly worse. This is consistent with the theoretical results obtained in Sections II and III.

\section{CONCLUSIONS}

We have established the close relationship among various short-time distributions and provided baseline results comparing the ASR performance of these alternative feature sets with the standard ASR front-end. Specifically, it was shown that 1) the difference between cepstrum ASR features derived from short-time averages of quadratic operators and the standard ASR front-end is a time-independent bias, provided that identical time-frequency tiling and narrowband filters are used in the ASR front-end and 2) $T F_{1}, T F_{2}$ and $T F_{3}$ are equivalent time-frequency representations when amplitude squared weighting is used $(\gamma=2)$, and can be expressed as the derivative of the spectral energy distribution $S$. The implications of these results for speech recognition were also discussed and experimentally verified. For matched training and testing conditions, ASR front-ends using cepstrum derived from averages of quadratic operators were shown to perform similarly to the standard ASR front end, while front-ends using first spectral moment features were shown to perform significantly worse.

\section{REFERENCES}

[1] L. Atlas and J. Fang, "Quadratic detectors for general nonlinear analysis of speech," in Proc. Int. Conf. Acoustics, Speech, Signal Processing, San Francisco, CA, Mar. 1992, pp. 9-12.

[2] J. F. Kaiser, "On a simple algorithm to calculate the "energy' of a signal," in Proc. Int. Conf. Acoustics, Speech, Signal Processing, Albuquerque, NM, Apr. 1990, pp. 381-384.

[3] P. Maragos, J. F. Kaiser, and T. F. Quatieri, "Energy separation in signal modulations with application to speech analysis," IEEE Trans. Signal Processing, vol. 41, pp. 3024-3051, Oct. 1993.

[4] P. Maragos and A. Potamianos, "Higher-order differential energy operators," IEEE Signal Processing Lett., vol. 2, Aug. 1995.

[5] R. J. McAulay and T. F. Quatieri, "Speech analysis/synthesis based on a sinusoidal representation," IEEE Trans. Acoust., Speech, Signal Processing, vol. 34, pp. 744-754, Aug. 1986. 
[6] K. K. Paliwal, "Spectral subband centroid features for speech recognition," in Proc. Int. Conf. Acoustics, Speech, Signal Processing, Seattle, WA, May 1998, pp. 617-620.

[7] J. W. Pitton, K. Wang, and B. H. Juang, "Time-frequency analysis and auditory modeling for automatic recognition of speech," Proc. IEEE, vol. 84, pp. 1199-1214, Sept. 1996.

[8] A. Potamianos and P. Maragos, "Applications of speech processing using an AM-FM modulation model and energy operators," in Proc. Eur. Signal Processing Conf., Edinburgh, U.K., Sept. 1994, pp. 1669-1672.

[9] —-, "Speech formant frequency and bandwidth tracking using multiband energy demodulation," J. Acoust. Soc. Amer., vol. 99, pp. 3795-3806, June 1996.

[10] —, "Speech analysis and synthesis using an AM-FM modulation model," Speech Commun., vol. 28, pp. 195-209, 1999.

[11] H. M. Teager, "Some observations on oral air flow during phonation," IEEE Trans. Acoust., Speech, Signal Processing, vol. ASSP-28, pp. 599-601, Oct. 1980.

[12] H. Tolba and D. O'Shaughnessy, "Automatic speech recognition based on cepstral coefficients and a mel-based discrete energy operator," in Proc. Int. Conf. Acoustics., Speech, Signal Processing, Seattle, WA, May 1998, pp. 973-976.

[13] G. Zhou, J. Hansen, and J. F. Kaiser, "Linear and nonlinear speech feature analysis for stress classification," in Int. Conf. Speech Language Processing, Sydney, Australia, Dec. 1998, pp. 840-843.

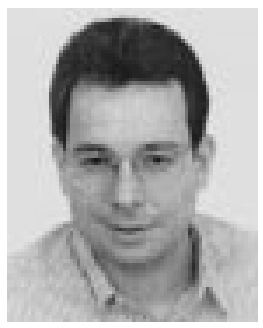

Alexandros Potamianos (M'92) received the Diploma degree in electrical and computer engineering from the National Technical University of Athens, Athens, Greece in 1990 and the the M.S. and $\mathrm{Ph} . \mathrm{D}$. degrees in engineering sciences from Harvard University, Cambridge, MA, in 1991 and 1995, respectively.

From 1991 to June 1993, he was a Research Assistant with Harvard Robotics Laboratory, Harvard University. From 1993 to 1995 , he was a Research Assistant with the Digital Signal Processing Laboratory, Georgia Institute of Technology, Atlanta. From 1995 to 1999, he was a Senior Technical Staff Member with the Speech and Image Processing Laboratory, AT\&T Shannon Laboratories, Florham Park, NJ. In February 1999, he joined the Multimedia Communications Laboratory, Bell Laboratories, Lucent Technologies, Murray Hill, NJ. He is also an adjunct Assistant Professor with the Department of Electrical Engineering, Columbia University, New York. He has authored or coauthored more than 30 papers in professional journals and conferences and holds three U.S. patents. His current research interests include speech processing, analysis, synthesis and recognition, dialogue and multimodal systems, nonlinear signal processing, natural language understanding, artificial intelligence, and multimodal child-computer interaction.

Dr. Potamianos has been a Member of the IEEE Signal Processing Society since 1992 and is currently a Member of the IEEE Speech Technical Committee.

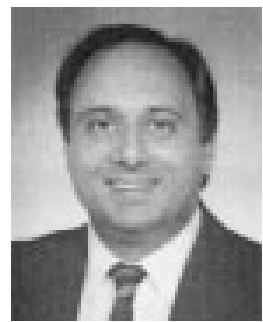

Petros Maragos (S'81-M'85-SM'91-F'95) received the Diploma degree in electrical engineering from the National Technical University of Athens, Athens, Greece, in 1980, and the M.S.E.E. and Ph.D. degrees in electrical engineering from the Georgia Institute of Technology, Atlanta, GA, in 1982 and 1985, respectively.

In 1985, he joined the faculty of the Division of Applied Sciences, Harvard University, Cambridge, MA, where he worked for eight years as Professor of electrical engineering, affiliated with the interdisciplinary Harvard Robotics Laboratory. He has also been a Consultant to several industry research groups including Xerox's research on document image analysis. In 1993, he joined the faculty of the School of Electrical and Computer Engineering at Georgia Tech. During parts of 1996-1998, he was on academic leave as a Senior Researcher with the Institute for Language and Speech Processing, Athens. In 1998, he joined the faculty of the National Technical University of Athens, where he is currently a Professor of electrical and computer engineering. His current research and teaching interests include the general areas of signal processing, systems theory, control, pattern recognition, and their applications to image processing and computer vision, and computer speech processing and recognition. He has served as Editorial Board Member for the Journal of Visual Communications and Image Representation.

Dr. Maragos has served as Associate Editor for the IEEE TRANSACTIONS ON ACOUSTICS, SPEECH, AND SignAl PROCESSING, and Guest Editor for the IEEE TRANSACTIONS ON IMAGE PROCESSING and member of two IEEE DSP committees. He was General Chairman for the 1992 SPIE Conference on Visual Communications and Image Processing, Co-Chairman for the 1996 International Symposium on Mathematical Morphology, and President of the International Society for Mathematical Morphology. His research work has received several awards, including a 1987 U.S. National Science Foundation Presidential Young Investigator Award; the 1988 IEEE Signal Processing Society's Paper Award for the paper "Morphological Filters"; the 1994 IEEE Signal Processing Society's Senior Award and the 1995 IEEE Baker Award for the paper "Energy Separation in Signal Modulations with Application to Speech Analysis" (co-recipient); and the 1996 Pattern Recognition Society's Honorable Mention Award for the paper "Min-Max Classifiers" (co-recipient). In 1995, he was elected Fellow of IEEE for his contributions to the theory and applications of nonlinear signal processing systems. 